Pacific Journal of Mathematic 


\title{
WEAK AND STRONG LIMITS OF SPECTRAL OPERATORS
}

\author{
WILliam G. BADE
}

The present paper is a contribution to the theory of spectral operators in Banach spaces developed by N. Dunford in [8] and [9]. A bounded operator $S$ is a spectral operator of scalar type if, roughly speaking, it has a representation

$$
S=\int_{\sigma(S)} \lambda E(d \lambda)
$$

where $E(\cdot)$ is a resolution of the identity similar to that possessed by a normal operator in Hilbert space. The initial problem we are concerned with is to find conditions under which a weak or strong limit of scalar type spectral operators is again in this class. The results are then applied to the study of certain weakly closed algebras of spectral operators.

Section 1 contains a brief summary of definitions and results from [8] and [9]. In $\$ 2$ conditions are found under which a strong limit of scalar type spectral operators is a scalar type spectral operator, the principal restriction imposed in the limiting operators being on the nature of their spectra. The operators need not commute.

Suppose that the underlying space $\mathcal{X}$ is reflexive. If $\mathcal{X}$ is an algebra generated by a bounded Boolean algebra $B$ of projections, then by a theorem of Dunford [9], each operator in $\mathfrak{U}$ is a scalar type spectral operator. We show (Theorem 4.1) that every operator in the weak closure $\mathfrak{X}$ of $\mathfrak{O}$ is a scalar type operator, and characterize $\mathfrak{B}$ as the algebra generated (in the uniform topology) by the strong closure of $\mathfrak{B}$. The principal tool used is the equivalence (due to Dunford [7]) of strong closure and lattice completeness for bounded Boolean algebras of projections. We give a new proof of this theorem.

The paper concludes with a characterization of the weakly closed algebra generated by a single scalar type spectral operator with real spectrum. Our proof of this theorem gives a more direct proof of the corresponding result of Segal [22] for Hilbert space.

Received February 8, 1954. The research contained in this paper was done under contract onr 609(04) with the Office of Naval Research.

Pacific J. Math. 4 (1954), 393-413 
1. Preliminaries. In this section we collect certain definitions and results, principally taken from [9].

Two projections in a Banach space $\mathfrak{X}$ are said to be ordered in their natural order, $E_{1} \leq E_{2}$ if $E_{1} E_{2}=E_{2} E_{1}=E_{1}$. This is equivalent to the conditions

$$
E_{1} \mathfrak{X} \subseteq E_{2} \mathfrak{X} \text { and }\left(I-E_{1}\right) \mathfrak{X} \supseteq\left(I-E_{2}\right) \mathfrak{X} \text {. }
$$

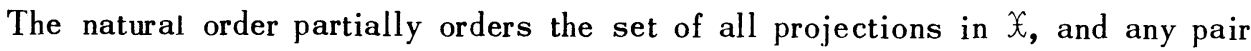
of commuting projections $E_{1}$ and $E_{2}$ has a least upper bound

$$
E_{1} \vee E_{2}=E_{1}+E_{2}-E_{1} E_{2}
$$

and greatest lower bound

$$
E_{1} \wedge E_{2}=E_{1} E_{2}
$$

If $\left\{E_{\alpha}\right\}$ is an arbitrary set of projections and $\nVdash$ admits the direct sum decomposition $\mathfrak{X}=m \oplus n$ where

$$
m=\overline{\operatorname{sp}}\left\{U_{a} E_{a} X\right\}, n=\Pi_{a}\left(I-E_{a}\right) X,
$$

then the projection with range $m$ defined by this decomposition is denoted by $\mathrm{V}_{\alpha} E_{\alpha}$ and is the least upper bound of the set $\left\{E_{\alpha}\right\}$. Correspondingly the greatest lower bound $\Lambda_{a} E_{a}$ with range $m_{1}$ is defined by the decomposition $x=m_{1} \oplus \eta_{1}$ where

$$
m_{1}=\bigcap_{a} E_{a} \mathfrak{X}, n_{1}=\overline{\mathrm{sp}}\left\{U_{\alpha}\left(I-E_{a}\right) X\right\},
$$

if it exists.

Throughout much of this paper we will be concerned with Boolean algebras of projections; that is, sets of commuting projections containing 0 and the identity $I$ which are Boolean algebras under the operations $E_{1} \vee E_{2}$ and $E_{1} \wedge E_{2}$. A Boolean algebra $B$ of projections is bounded if there is a constant $M$ such that $|E| \leq M$ for $E \in B$. $B$ is complete if it contains $\vee_{\alpha} E_{\alpha}$ and $\wedge_{\alpha} E_{\alpha}$ for every subset $\left\{E_{\alpha}\right\} \subseteq B$. We remark that $\mathscr{B}$ may be complete as a lattice but not complete as a Boolean algebra of projections in $X$ in the present sense.

Let $₹$ be a $\sigma$-field of subsets of a set $\Omega$. A homomorphic map $E(\cdot)$ of $₹$ onto a bounded Boolean algebra of projections in $\mathfrak{X}$ will be called a spectral measure. Thus 


$$
\left.\begin{array}{l}
E(\sigma \cup \delta)=E(\sigma) \vee E(\delta), E(\sigma \cap \delta)=E(\sigma) \wedge E(\delta), \\
E(\Omega)=I, E\left(\sigma^{\prime}\right)=I-E(\sigma),|E(\sigma)| \leq M
\end{array}\right\} \sigma, \delta \in \widetilde{\mho} .
$$

The set function $x^{*} E(\cdot) x, x \in \mathfrak{X}, x^{*} \in \mathfrak{X}^{*}$, satisfies (see proof of [9, Theorem 17])

$$
\operatorname{var} x^{*} E(\cdot) x \leq 4 M|x|\left|x^{*}\right| \text {. }
$$

The spectral measure $E(\cdot)$ is countably additive if $x^{*} E(\cdot) x$ is countably additive for each $x \in \mathfrak{X}, x^{*} \in \mathfrak{X}^{*}$. Countable additivity of $E(\cdot)$ implies that the vector valued set functions $E(\cdot) x$ are countably additive for $x \in \mathfrak{X}[8$, page 579].

If $F(\cdot)$ is a spectral measure in the conjugate space $\mathfrak{X}^{*}$ of $\mathfrak{X}$ we say $F(\cdot)$ is $(X)$-countably additive if $F(\cdot) x^{*} x$ is countably additive for all $x^{*} \in X^{*}$ and $x \in \mathfrak{X}$.

We will need a notion of integration of scalar functions with respect to a spectral measure [9, Lemma 6]. Let $E(\cdot)$ on $(\Omega, ₹)$ be either a countably additive spectral measure in $X$ or an $(X)$-countably additive spectral measure in $\mathcal{X}^{*}$. Then for $f$ an essentially bounded measurable function on $\Omega$, the integral $\int_{\Omega} f(\omega) E(d \omega)$ is defined as the limit

$$
\int_{\Omega} f(\omega) E(d \omega)=\lim _{n \rightarrow \infty} \int_{\Omega} f_{n}(\omega) E(d \omega)
$$

in the uniform operator topology, where the functions

$$
f_{n}(\omega)=\sum \alpha_{i n} k_{\sigma_{i n}}(\omega)
$$$$
n=1,2, \cdots,
$$

form a sequence of finite linear combinations of characteristic functions of disjoint sets $\sigma_{i n} \in \mathcal{F}$ converging uniformly to $f$ on $\Omega$ and

$$
\int_{\Omega} f_{n}(\omega) E(d \omega)=\sum \alpha_{i n} E\left(\sigma_{i n}\right) .
$$

This integral satisfies

$$
\frac{1}{4 M} \operatorname{ess} \Omega^{\inf }|f(\omega)| \leq\left|\int_{\Omega} f(\omega) E(d \omega)\right| \leq 4 M \text { ess } \Omega \text { sup }|f(\omega)| .
$$

A countably additive spectral measure on the Borel sets of the complex plane 
is called a resolution of the identity. A bounded operator $T$ in $X$ is called a spectral operator of scalar type if there is a resolution of the identity $E(\cdot)$ such that

$$
\left.\begin{array}{l}
E(\mu) T=T E(\mu) \\
\sigma(T ; E(\mu) X) \subseteq \bar{\mu}
\end{array}\right\} \mu \in \text { Borel sets }
$$

and

$$
T=\int_{\sigma(T)} \lambda E(d \lambda)
$$

Here $\sigma(T ; E(\mu) \mathcal{X})$ is the spectrum of the restriction of $T$ to the range of $E(\mu)$. In exactly the same way we have the notions of an $(X)$-countably additive resolution of the identity and a scalar type spectral operator of class $(X)$ in $\mathfrak{X}^{*}$. In either case $E(\cdot)$ is unique and $E(\sigma(T))=I$. Moreover if $F(\cdot)$ is a countably additive $((\mathfrak{X})$-countably additive $)$ spectral measure on $(\Omega, \mathfrak{F})$, the operator

$$
S(f)=\int_{\Omega} f(\omega) F(d \omega)
$$

defined by (1.3) is a spectral operator of scalar type (scalar type and class $(X))$ whose resolution of the identity $E(\cdot ; S(f))$ is given by

$$
E(\sigma ; S(f))=F\left(f^{-1}(\sigma)\right), \sigma \in \text { Borel sets. }
$$

Finally we will need the following specialization of a theorem of Dunford [9, Theorem 17].

1.1 ThEOREM. Let $B$ be a bounded Boolean algebra of projections in a reflexive space and let $\mathfrak{A}$ be the algebra generated by $\mathfrak{B}$ in the uniform operator topology. If $\mathfrak{M}$ denotes the compact Hausdorff space of maximal ideals in $\mathfrak{A}$, then $\mathfrak{A}$ is equivalent to $C(\mathfrak{M})$ under a topological and algebraic isomorphism $S$. There is a spectral measure $E(\cdot)$ defined on the Baire sets of $M$ such that if $f \in C(\mathbb{M})$, then

$$
S(f)=\int_{\mathbb{M}} f(m) E(d m)
$$

1.2 REMARKS. By the discussion above each operator in $\mathscr{U}$ is a scalar type 
spectral operator. It is easily seen that $\mathbb{M}$ may be identified with the Stone representation space of the Boolean algebra $B$. Since $\mathbb{M}$ is totally disconnected, the class $\mathcal{F}$ of Baire sets of $M$ is generated by the open and closed sets. Thus $\{E(\sigma) \mid \sigma \in \mathcal{F}\}$ is an extension of $B$ and lies in the strong closure of $B$ by the strong countable additivity of $E(\cdot) x, x \in \mathfrak{X}$.

2. Strong limits of operators with restricted spectra. In this section we determine conditions under which a limit in the strong operator topology of scalar type spectral operators is again in this class. The principal restriction imposed on the limiting operators is on the distribution of their spectra. For application in later sections our principal result (Theorem 2.3) is stated in terms of Moore-Smith convergence, or convergence of nets in the terminology of Kelley [15]. We recall that the strong operator topology for $B(\mathfrak{X})$ is generated by neighborhoods of the form

$$
N\left(T_{0} ; x_{1}, \cdots, x_{n}, \epsilon\right)=\left\{T||\left(T-T_{0}\right) x_{i} \mid<\epsilon, i=1, \cdots, n\right\},
$$

the weak operator topology by neighborhoods of the form

$$
N\left(T_{0} ; x_{1}, \cdots, x_{n}, x_{1}^{*}, \ldots, x_{n}^{*}, \epsilon\right)=\left\{T|| x_{i}^{*}\left(T-T_{0}\right) x_{i} \mid<\epsilon, i=1, \cdots, n\right\} .
$$

A net $\left\{T_{\alpha}\right\}, \alpha \in A$, converges strongly to $T \in B(X)$ if

$$
\lim _{\alpha} T_{\alpha} x=T x
$$

It converges weakly to $T$ if

$$
\lim _{\alpha} x^{*} T_{\alpha} x=x^{*} T x
$$$$
x \in \mathfrak{X}, x^{*} \in \mathfrak{X}^{*} \text {. }
$$

If $V$ is an unbounded closed subset of the complex plane we denote by $C_{\infty}(V)$ the $B$-space of complex valued continuous functions on $V$ which vanish at infinity (A function $f$ vanishes at infinity on $V$ if given $\epsilon>0$ there is a number $K$ with $|f(\lambda)|<\epsilon$ if $\lambda \in V,|\lambda|>K$.) If $V$ is bounded we let $C_{\infty}(V)=$ $C(V)$.

2.1. Definition. A closed nowhere dense set $V$ in the complex plane will be called an $R$-set if the set of functions

$$
\left\{f \mid f(\lambda)=\frac{1}{\mu-\lambda}, \mu \notin V\right\}
$$


is a fundamental in $C_{\infty}(V)$.

It is easily shown that $V$ is an $R$-set if and only if rational functions are dense in $C_{\infty}(V)$. To approximate a rational function $q$ in $C_{\infty}(V)$ by linear combinations of functions of the prescribed type when $V$ is unbounded, one approximates by Riemann sums the integral in the representation

$$
q(\lambda)=\frac{1}{2 \pi i} \int_{C} \frac{q(\mu)}{\mu-\lambda} d \mu, \quad \lambda \in V \text {. }
$$

Here $C$ is a clockwise contour consisting of small circles exterior to $V$ which contain the poles of $q$. We leave to the reader the fact that the approximation may be made uniform on $V$. The case where $V$ is bounded is treated by a similar argument.

The characterization of $R$-sets is apparently an unsolved problem of approximation theory. It is known that not every closed nowhere dense set is an $R$-set. The most important example of an $R$-set is, of course, the real line. That $R$-sets form an extensive class of sets is shown by the following lemma.

2.2. LEMMA. In order for a closed nowhere dense set $V$ to be an $R$-set it is sufficient either that $V$ has plane measure zero or that $V$ does not separate the plane.

The case that $V$ is bounded follows from important theorems of approximation theory. By a theorem of Lavrentieff [16] (see also Mergelyan [18]) polynomials are dense in $C(V)$ if $V$ does not separate the plane. Hartogs and Rosenthal [12] have shown that rational functions are dense in $C(V)$ if $V$ has plane measure zero. If $V$ is unbounded let $V_{1}=V \cup\{\infty\}$ have the usual topology as a subset of the complex sphere. If $\beta \notin V$ the mapping $\Phi$ defined by $\Phi(\lambda)=$ $(\beta-\lambda)^{-1}$ maps $V_{1}$ homeomorphically onto a closed and bounded nowhere dense set $W$ containing zero. If $f \in C_{\infty}(V)$ then $\phi(z)=f\left(\Phi^{-1}(z)\right)$ is in $C(W)$ and vanishes at zero. Moreover $\phi$ is rational if and only if $f$ is rational, and $W$ does not separate the plane or has measure zero if and only if $V$ has the same property.

We now suppose that $\left\{T_{\alpha}\right\}, \alpha \in A$, is a net of bounded scalar type spectral operators with $\lim _{\alpha} T_{\alpha} x=T x, x \in \mathfrak{X}, T \in B(\mathfrak{X})$. The operators $T_{\alpha}$ need not commute or be uniformly bounded in norm. We examine the spectral properties of $T$ under two assumptions.

(A). If $E_{\alpha}(\cdot)$ denotes the resolution of the identity for $T_{\alpha}$, then there is a constant $M$ such that $\left|E_{\alpha}(\sigma)\right| \leq M, \alpha \in A, \sigma \in$ Borel sets. 
(B). There is a fixed closed (possibly unbounded) $R$-set $V$ with $\sigma\left(T_{\alpha}\right) \subseteq$ $V, \alpha \in A$.

2.3. THE OREM. If $T \in B(\mathfrak{X})$ is the strong limit of a net $\left\{T_{a}\right\}$ of scalar type spectral operators satisfying conditions (A) and (B), then $T^{*}$ is a scalar type spectral operator in $\mathfrak{X}^{*}$ of class $(\mathfrak{X})$. If $\mathfrak{X}$ is reflexive, $T$ is a scalar type spectral operator in $\mathfrak{X}$.

It should be remarked that for applications in later sections we will need only the case that $V$ is the real line. The method of proof is a straightforward extension of that used by Stone in [23] to prove the spectral theorem in Hilbert space. The proof will require two lemmas.

2.4. Lemma. If $\lambda \notin V$ then $\lambda \in \rho(T)$,

$$
\left|R(\lambda ; T)^{\prime}\right| \leq 4 M d(\lambda, V)^{-1}
$$

(where $d(\lambda, V)=$ dist. $(\lambda, V))$, and

$$
\lim _{\alpha} R\left(\lambda ; T_{\alpha}\right) x=R(\lambda ; T) x,
$$

Proof. Since

$$
R\left(\lambda, T_{\alpha}\right)=\int_{\sigma\left(T_{\alpha}\right)}(\lambda-\mu)^{-1} E_{\sigma}(d \mu)
$$

we have from (1.4)

$$
\left|\left(\lambda I-T_{a}\right) x\right| \geq \frac{|x| d(\lambda, V)}{4 M}, \quad \alpha \in A, x \in X,
$$

from which it follows that

$$
|(\lambda I-T) x| \geq \frac{|x| d(\lambda, V)}{4 M}, \quad x \in \mathfrak{X} .
$$

The last conclusion follows from the identity

$$
R\left(\lambda ; T_{\alpha}\right) x-R(\lambda ; T) x=R\left(\lambda ; T_{\alpha}\right)\left(T-T_{\alpha}\right) R(\lambda ; T) x .
$$

2.5. Lemma. Given $x \in \mathfrak{X}, x^{*} \in \mathfrak{X}^{*}$, there is a unique measure $\rho\left(\cdot ; x^{*}, x\right)$, bilinear in $x$ and $x^{*}$, which satisfies 


$$
x^{*} R(\lambda ; T) x=\int_{V} \frac{1}{\lambda-\mu} \rho\left(d \mu ; x^{*}, x\right), \quad \lambda \notin V .
$$

Moreover

$$
\operatorname{var}\left(\rho\left(\cdot ; x^{*} x\right)\right) \leq 4 M|x|\left|x^{*}\right|
$$

Proof. By (1.2)

$$
\operatorname{var} x^{*} E_{\sigma}(\cdot) x \leq 4 M|x|\left|x^{*}\right| \text {. }
$$

Thus the set of measures $\left\{x^{*} E_{a}(\cdot) x\right\}, \alpha \in A$, is a net in the closed sphere $S$ about the origin of radius $4 M|x|\left|x^{*}\right|$ in the space $R(V)$ of regular measures on the Borel sets of $V$. Since $R(V)$ is the conjugate space of $C_{\infty}(V)$, the set $S$ is compact in the $w^{*}$-topology $[\mathbf{1}]$; that is, the topology generated by neighborhoods of the form

$$
\begin{aligned}
& N\left(\theta_{0} ; f_{1}, \ldots, f_{n}, \epsilon\right)= \\
& \quad\left\{\theta|\theta \in R(V),| \int_{V} f_{i}(\lambda) \theta(d \lambda)-\int_{V} f_{i}(\lambda) \theta_{0}(d \lambda) \mid<\epsilon, i=1, \cdots, n\right\},
\end{aligned}
$$

where $f_{1}, \cdots, f_{n} \in C_{\infty}(V)$. It follows $[15]$ that the net $\left\{x^{*} E_{\alpha}(\cdot) x\right\}$ has a cluster point $\rho\left(\cdot ; x^{*}, x\right)$; that is, given $\alpha_{0} \in A$, every neighborhood of $\rho$ contains a measure $x^{*} E_{\alpha}(\cdot) x$ for some $\alpha \geq \alpha_{0}$. In particular if $\lambda \notin V, \epsilon>0$, and $\alpha_{0} \in A$, then

$$
\begin{aligned}
& \left|\int_{V} \frac{1}{\lambda-\mu} x^{*} E_{\alpha}(d \mu) x-\int_{V} \frac{1}{\lambda-\mu} \rho\left(d \mu ; x^{*}, x\right)\right| \\
& =\left|x^{*} R\left(\lambda ; T_{\alpha}\right) x-\int_{V} \frac{1}{\lambda-\mu} \rho\left(d \mu ; x^{*}, x\right)\right|<\epsilon
\end{aligned}
$$

for some $a \geq \alpha_{0}$. By Lemma 2.4,

$$
\lim _{\alpha} x^{*} R\left(\lambda ; T_{\alpha}\right) x=x^{*} R(\lambda ; T) x .
$$

Thus

$$
x^{*} R(\lambda ; T) x=\int_{V} \frac{1}{\lambda-\mu} \rho\left(d \mu ; x^{*}, x\right), \quad \lambda \notin V, x^{*} \in \mathfrak{X}^{*}, x \in \mathfrak{X} .
$$

The uniqueness of $\rho\left(\cdot ; x^{*}, x\right)$ and its bilinearity in $x$ and $x^{*}$ follow from the fact $V$ is an $R$-set. 
To continue the proof of the theorem, we now extend the measure $\rho\left(\cdot ; x^{*}, x\right)$ on $V$ to all Borel sets of the plane in the obvious way. Since for any Borel set $e$,

$$
\left|\rho\left(e ; x^{*}, x\right)\right| \leq 4 M\left|x^{*}\right||x|
$$

there is a unique operator $A(e)$ in $X^{*}$ satisfying $|A(e)| \leq 4 M$ and

$$
\rho\left(e ; x^{*}, x\right)=A(e) x^{*} x, \quad x^{*} \in \mathfrak{X}^{*}, x \in \mathfrak{X} .
$$

It will now be shown that the family $\{A(\cdot)\}$ is a resolution of the identity for $T^{*}$. Let $\nu \notin V$. Then

$$
R\left(\nu ; T^{*}\right) A\left(e_{0}\right) x^{*} x=\int_{e_{0}} \frac{1}{\nu-\mu} A(d \mu) x^{*} x
$$

for each Borel set $e_{0}$; for the equation

$$
\int_{V} \frac{R\left(\nu ; T^{*}\right)}{\lambda-\mu} A(d \mu) x^{*} x=R\left(\nu ; T^{*}\right) R\left(\lambda ; T^{*}\right) x^{*} x=\int_{V} \frac{\theta(d \mu)}{\lambda-\mu},
$$

where

$$
\theta(e)=\int_{e} \frac{A(d \mu) x^{*} x}{\nu-\mu}
$$

is valid for every $\lambda \notin V, \lambda \neq \nu$. Since the corresponding functions $(\lambda-\mu)^{-1}$ are fundamental in $C_{\infty}(V)$, formula (2.1) follows from equating the measures in (2.2). However,

$$
R\left(\nu ; T^{*}\right) A\left(e_{0}\right) x^{*} x=\int_{V} \frac{1}{\nu-\mu} A(d \mu) A\left(e_{0}\right) x^{*} x,
$$

and the same uniqueness argument for the measure yields

$$
A\left(e_{0}\right) A\left(e_{1}\right)=A\left(e_{0} \cap e_{1}\right)
$$

for arbitrary Borel sets $e_{0}$ and $e_{1}$. Hence $A(e)$ is a projection. In view of the countable additivity of $A(\cdot) x^{*} x$, it remains to show that $\sigma\left(T^{*}\right)=I$ and $\sigma\left(T^{*}, A(e) \mathfrak{X}\right) \subseteq \bar{e}$ for arbitrary $e$. The second statement follows from formula (2.1) since $R\left(\nu ; T^{*}\right) A(e) x^{*} x$ has a unique analytic continuation to all of $e^{\prime}$, because $\sigma\left(T^{*}\right) \cap \bar{e}$ is nowhere dense. To prove the first statement let $e_{0}$ be 
any compact subset of $V-\sigma\left(T^{*}\right)$. Since

$$
R\left(\lambda ; T^{*}\right) A\left(e_{0}\right)=A\left(e_{0}\right) R\left(\lambda ; T^{*}\right) \text { for } \lambda \in \rho\left(T^{*}\right),
$$

$A\left(e_{0}\right)$ commutes with $T^{*}$. Thus

$$
\sigma\left(T^{*}, A\left(e_{0}\right) X^{*}\right) \subseteq \sigma\left(T^{*}\right)
$$

But again $R\left(\lambda ; T^{*}\right) A\left(e_{0}\right) x^{*} x$ has a unique analytic continuation to $e_{0}^{\prime}$, from which it follows that $A\left(e_{0}\right)=0$, and hence $A\left(V-\sigma\left(T^{*}\right)\right)=0$ as $V-\sigma\left(T^{*}\right)$ is the union of an ascending sequence of compact sets. Finally if $C$ is any contour enclosing the bounded set $\sigma\left(T^{*}\right)$,

$$
x^{*} x=\int_{\sigma\left(T^{*}\right)}\left\{\frac{1}{2 \pi i} \int_{C} \frac{1}{\lambda-\mu} d \lambda\right\} A(d \mu) x^{*} x=A\left(\sigma\left(T^{*}\right)\right) x^{*} x,
$$

showing $A\left(\sigma\left(T^{*}\right)\right)=I$. If $氏$ is reflexive the projections $E(e)=A^{*}(e)$ form a resolution of the identity for $T$ in $\mathfrak{X}$. This completes the proof.

2.6. THEOREM. Let a net $\left\{T_{\alpha}\right\}, \alpha \in A$, of bounded scalar type spectral operators satisfying conditions (A) and (B) converge strongly to a bounded scalar type spectral operator $T$. Let $h$ be a bounded Borel function on $V$ with set $K$ of discontinuities. If $E(\bar{K})=0$ where $E(\cdot)$ is the resolution of the identity for $T$, then $h\left(T_{a}\right)$ converges strongly to $h(T)$.

Proof. We consider first the case that $h \in C_{\infty}(V)$. By Lemma 2.4, $R(\lambda ; T)$, $\lambda \notin V$, is the strong limit of $R\left(\lambda ; T_{\alpha}\right)$, and hence $\lim _{\alpha} g\left(T_{\alpha}\right)=g(T)$ strongly for $g$ in a dense subset of $C_{\infty}(V)$. If $|h-g|<\epsilon$, then

$$
\begin{aligned}
\left|h\left(T_{\alpha}\right) x-h(T) x\right| & \leq\left|\int_{V}(h(\lambda))-g(\lambda) E_{\alpha}(d \lambda) x\right| \\
+\mid \int_{V} & (h(\lambda)-g(\lambda)) E(d \lambda) x|+| g\left(T_{\alpha}\right) x-g(T) x \mid \\
& \leq 8 M \in|x|+\left|g\left(T_{\alpha}\right) x-g(T) x\right|, \quad x \in \mathfrak{X},
\end{aligned}
$$

from which the conclusion follows for $h \in C_{\infty}(V)$. In the case $h$ is a bounded Borel function whose set $K$ of discontinuities satisfies $E(\bar{K})=0$, choose $g \in C_{\infty}(V)$ such that $g(\lambda)=0, \lambda \in K$, and $g(\lambda)>0$ for $\lambda \in V-\bar{K}$. The function $g h$ is in $C_{\infty}(V)$. Moreover, the range of $g(T)$ is dense in $X$; for given $x \in X$ and $\epsilon>0$ there is a closed subset $\sigma$ of $V$ disjoint from $\bar{K}$ such that 
$|x-E(\sigma) x|<\epsilon$. Then

$$
E(\sigma) x=g(T) y \text { where } y=\int_{\sigma} \frac{E(d \lambda) x}{g(\lambda)} .
$$

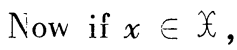

$$
\begin{aligned}
& \left|h\left(T_{\alpha}\right) g(T) x-h(T) g(T) x\right| \\
& \leq\left|h\left(T_{\alpha}\right) g(T) x-h\left(T_{\alpha}\right) g\left(T_{\alpha}\right) x\right|+\left|(h g)\left(T_{\alpha}\right) x-(h g)(T) x\right| \\
& \leq 4 M \text { ess } \sup _{\lambda \in V}|h(\lambda)| \cdot\left|g(T) x-g\left(T_{\alpha}\right) x\right|+\left|(h g)\left(T_{\alpha}\right)-(h g)(T) x\right| .
\end{aligned}
$$

By the previous case $g\left(T_{\alpha}\right)$ and $(h g)\left(T_{\alpha}\right)$ converge strongly to $g(T)$ and $(h g)(T)$. Thus $\lim _{\alpha} h\left(T_{\alpha}\right) y=h(T) y$ for $y$ in a dense set. Since the $h\left(T_{a}\right)$ are uniformly bounded, $h\left(T_{\alpha}\right)$ converges strongly to $h(T)$.

Theorem 2.6 generalizes a theorem of Kaplansky [14] for the case that the $T_{\alpha}$ are self adjoint operators on Hilbert space and $\bar{K} \cap \sigma(T)=0$. The present theorem contains a result of Rellich [21] that if $\left\{T_{n}\right\}$ is a sequence of self adjoint operators converging strongly to $T$, then

$$
\lim _{n \rightarrow \infty} E_{n}((-\infty, \lambda]) x=E((-\infty, \lambda]) x, \quad x \in \mathfrak{X}
$$

for each $\lambda$ not in the point spectrum of $T$.

3. Bounded Boolean algebras of projections. It is natural to ask when a Boolean algebra $B$ of projections may be embedded in a complete Boolean algebra of projections. Under the assumptions that $X$ is reflexive and $B$ is bounded, Dunford in [7] constructs the projection $V_{\alpha} E_{a}$ corresponding to any subset $\left\{E_{a}\right\} \subseteq B$, and states the theorem that the least complete Boolean algebra of projections containing $B$ is the closure of $B$ in the strong operator topology. In this section we will give a proof of Dunford's theorem by showing first that the strong closure of $B$ (denoted by $\bar{B} s$ ) is complete. It will then be required to show that a complete bounded Boolean algebra of projections is strongly closed. Actually we will show it contains every projection in the weakly (equivalently, strongly) closed algebra which it generates. This stronger result will be needed in $\$ 4$.

The proofs will require the following lemma on monotone nets of projections.

3.1. LеммA. Let $\left\{E_{\alpha}\right\}, \alpha \in A$, be a net of projections in a reflexive space 
$¥$ satisfying $\left|E_{a}\right| \leq M, \alpha \in A$. If $E_{\alpha} \leq E_{\beta}$ whenever $\alpha \leq \beta$, then $\lim _{\alpha} E_{a}$ exists in the strong operator topology and $\lim _{\alpha} E_{\alpha}=\mathrm{V}_{\alpha} E_{\alpha}$. Correspondingly if $E_{\beta} \leq E_{\alpha}$ whenever $\alpha \leq \beta$, then $\lim _{\alpha} E_{\alpha}=\Lambda_{\alpha} E_{\alpha}$ in the strong operator topology.

This result is due to Lorch [17] for the case of monotone sequences. A proof of the general case has been given by J. Y. Barry [3].

3.2. THEOREM. If $\mathfrak{B}$ is a bounded Boolean algebra of projections in a reflexive space, then $\overline{B^{s}}$ is a complete bounded Boolean algebra of projections containing $\mathfrak{B}$.

Proof. Clearly $|E| \leq M$ if $E \in \overline{B^{s}}$. If $E, E_{1}, F$ and $F_{1}$ are in $\mathscr{B}, \mid(E-$ $\left.E_{1}\right) x \mid<\epsilon$, and $\left|\left(F-F_{1}\right) x\right|<\epsilon$, then

$$
\left|\left(E F-E_{1} F_{1}\right) x\right| \leq\left|\left(E F-E F_{1}\right) x\right|+\left|\left(E F_{1}-E_{1} F_{1}\right) x\right| \leq 2 M \epsilon .
$$

Thus the mapping $[E, F] \longrightarrow E F$ is a continuous map of $B \times B \rightarrow B(X)$ in the strong operator topology. Thus $\bar{B} s$ is a bounded Boolean algebra of projections. If $\Re_{0}$ is any subset of $\bar{B}^{s}$, let $\Sigma$ be the family of all finite subsets of $\mathfrak{B}_{0}$, directed by inclusion. If $\sigma=\left\{E_{1}, \cdots, E_{n}\right\} \subseteq \mathbb{B}_{0}$ let $E_{\sigma}=E_{1} \vee E_{2} \vee \cdots \vee E_{n}$. The net $\left\{E_{\sigma}\right\}, \sigma \in \Sigma$, is monotone in the natural order of projections. By Lemma 3.1, we have

$$
\lim _{\sigma} E_{\sigma}=\mathrm{V}_{\sigma} E_{\sigma} \in \overline{B^{s}} s
$$

The next lemma is an extension of a result of Dixmier [5] for Hilbert space ( see also Michael [19]). The proof is similar, but we give it for completeness.

3.3. Lemma. If $\mathfrak{X}$ is $B$-space, a convex subset of $B(\mathfrak{X})$ has the same closure in the weak operator topology as it does in the strong operator topology.

Proof. Under either the weak or the strong operator topology $B(X)$ is a locally convex linear topological space. In view of the separation theorem for convex sets [4] it is enough to show that these two spaces have the same continuous linear functionals; or, since the strong topology is stronger than the weak, that a functional continuous in the strong topology is continuous in the weak topology. If $\theta$ is continuous in the strong topology, there is a finite subset $\left\{x_{1}, \cdots, x_{n}\right\}$ of $X$ and an $\epsilon>0$, such that $\left|T x_{i}\right|<\epsilon, i=1, \cdots, n, T \in B(X)$, implies $|\theta(T)|<1$. Let $\lesssim$ be the Banach space of $n$-tuples $\zeta=\left[z_{1}, \ldots, z_{n}\right]$, $z_{i} \in \mathfrak{X}$ with norm $|\zeta|=\max _{1} \leq i \leq n\left|z_{i}\right|$. If $\Phi$ is the mapping of $B(\mathfrak{X})$ into 
3 defined by $\Phi(T)=\left[T x_{1}, \ldots, T x_{n}\right]$, it is easily seen that the functional $f_{0}$ on $\Phi(B(\mathfrak{X}))$ defined by $f_{0}(\zeta)=\theta(T)$ is well defined and continuous. If $f$ is a continuous extension of $f_{0}$ to all of $B$, then $f$ has the form

$$
f\left(\left[z_{1}, \cdots, z_{n}\right]\right)=\sum_{i=1}^{n} x_{i}^{*} z_{i}
$$

where $x_{i}^{*} \in \mathfrak{X}^{*}$. Consequently $\theta(T)=f(\Phi(T))$ has the form

$$
\theta(T)=\sum_{i=1}^{n} x_{i}^{*} T x_{i} .
$$

It follows that $\theta$ is continuous in the weak operator topology.

3.4. THEOREM. ${ }^{1}$ A complete bounded Boolean algebra of projections in a reflexive space contains every projection in the weakly closed algebra it generates.

Proof. Let $\mathcal{A}$ be the algebra generated by $\mathscr{B}$ in the uniform operator topology, and let $\overline{\mathfrak{Q}}^{w}$ be the closure of $\mathfrak{X}$ in the weak operator topology. Since $\overline{\mathfrak{V}} w$ is an algebra, it is the weakly closed algebra generated by $\mathfrak{B}$. Moreover, $\overline{\mathfrak{L}}^{w}=\overline{\mathfrak{Q}}^{s}$ by Lemma 3.3. Let $F^{2}=F, F \in \overline{\mathfrak{M}}^{s}$. The proof that $F \in \Re$ will be made by showing that to each pair $(y, z)$ where $y \in M=F \mathfrak{X}$ and $z \in \eta=(I-F) \mathfrak{X}$ there can be associated a projection $E_{y z} \in B$ such that $E_{y z} y=y=F y$, and $E_{y z} z=0=F z$. For if this is granted, the projection

$$
E=\wedge_{z} \in \eta \vee_{y \in m} E_{y z}
$$

is in $B$ since $B$ is complete. If $x_{0} \in X, x_{0}=y_{0}+z_{0}, y_{0} \in M, z_{0} \in \eta$, then $\vee_{y \in m} E_{y z} y_{0}=y_{0}$ for each $z \in \eta$, and $\vee_{y \in M} E_{y z_{0}} z_{0}=0$. Thus $E y_{0}=y_{0}$, $E z_{0}=0$ and $E=F$.

We now construct the projections $E_{y z}$. It should be remarked that the construction uses only the fact that $B$ is $\sigma$-complete. Let $y$ and $z$ be fixed elements of $m$ and $n$ respectively. Then since $F \in \overline{\mathfrak{X}}^{s}$, elements $A_{n} \in \mathfrak{U}$ may be selected such that

$$
\left|y-A_{n} y\right|<1 / 2^{n},\left|A_{n} z\right|<1 / 2^{n},
$$$$
n=1,2, \cdots,
$$

${ }^{1}$ This theorem does not answer the question: if a sequence $\left\{E_{n}\right\} \subseteq B$ converges weakly to a projection $F$, does $\left\{E_{n}\right\}$ converge strongly to $F$ ? 
and each $A_{n}$ is a finite linear combination of disjoint projections $\left\{E_{1}, \ldots, E_{s_{n}}\right\}$ in $\mathscr{B}$. It is now convenient to use the fact (Theorem 1.1) that $\mathscr{U}$ is equivalent to $C(\mathbb{M})$ where $\mathbb{M}$ is the space of maximal ideals in 2 . Thus $A_{n}=S\left(f_{n}\right)$ where $f_{n}$ is a finite linear combination of characteristic functions of disjoint open and closed sets, and $A_{n}=\int_{\mathfrak{M}} f_{n}(m) E(d m)$, where the integral is that of $\xi 1$. Let $\epsilon$ be an arbitrary positive number and

$$
\sigma_{n \epsilon}=\left\{m|| f_{n}(m) \mid>\epsilon\right\} .
$$

If $E\left(\sigma_{n \epsilon}\right)$ is the projection corresponding to $\sigma_{n \epsilon}$, the remainder of the proof consists of showing we may take

$$
E_{y z}=\mathrm{V}_{\epsilon>0} \wedge_{n=1}^{\infty} \bigvee_{i=n}^{\infty} E\left(\sigma_{i \epsilon}\right)
$$

Let

$$
E_{n \epsilon}=\vee_{i=n}^{\infty} E\left(\sigma_{i \epsilon}\right), E_{0 \epsilon}=\Lambda_{n=1}^{\infty} E_{n \epsilon} .
$$

Since the sequence $\left\{E_{n \epsilon}\right\}$ is monotone decreasing,

$$
\lim _{n \rightarrow \infty} E_{n \epsilon} y=E_{0 \epsilon} y
$$

by Lemma 3.1. Defining

$$
A_{n \epsilon}=\int_{\sigma_{n \epsilon}} f_{n}(m) E(d m)
$$

we have

$$
\begin{aligned}
\left|E_{n \epsilon} y-y\right| & \leq\left|E_{n \epsilon}\left(y-A_{n} y\right)\right|+\left|A_{n \epsilon} y-A_{n} y\right|+\left|A_{n} y-y\right| \\
& <\frac{(M+1)}{2^{n}}+\left|\int_{\sigma_{n \epsilon}^{\prime}} f_{n}(m) E(d m) y\right| \leq \frac{(M+1)}{2^{n}}+4 M \epsilon|y| .
\end{aligned}
$$

Thus

$$
\left|E_{0 \epsilon} y-y\right| \leq 4 M \epsilon|y| \text {. }
$$




$$
E_{n \epsilon} z=\lim _{p \rightarrow \infty} \bigvee_{i=n}^{p} E\left(\sigma_{i \epsilon}\right) z
$$

by Lemma 3.1. But

$$
\begin{aligned}
&\left|\mathrm{V}_{i=1}^{p} E\left(\sigma_{i \epsilon}\right) z\right|=\mid\left\{E\left(\sigma_{n \epsilon}\right)+E\left(\sigma_{(n+1) \epsilon}\right)\left(I-E\left(\sigma_{n \epsilon}\right)\right)\right. \\
&\left.+\cdots+E\left(\sigma_{p \epsilon}\right)\left(I-E\left(\bigcup_{j=n}^{p-1} \sigma_{j \epsilon}\right)\right)\right\} z\left|\leq N \sum_{i=n}^{p}\right| E\left(\sigma_{i \epsilon}\right) z \mid .
\end{aligned}
$$

Since by (1.4)

$$
\left|A_{i \epsilon} z\right|=\left|A_{i} E\left(\sigma_{i \epsilon}\right) z\right| \geq \frac{\epsilon\left|E\left(\sigma_{i \epsilon}\right) z\right|}{4 M}
$$

we have

$$
\left|E\left(\sigma_{i \epsilon}\right) z\right| \leq \frac{4 M^{2}}{\epsilon 2^{i}}, \quad i \geq n
$$

Thus

$$
\left|E_{n \epsilon} z\right|<\frac{4 M^{3}}{\epsilon 2^{n-1}}
$$

and

$$
E_{0 \epsilon} z=0
$$

by Lemma 3.1. If $0<\delta<\epsilon$ then $E_{0 \epsilon} \leq E_{0 \delta}$; so if $\epsilon_{n}=1 / n$ and

$$
E_{0}=\mathrm{V}_{n=1}^{\infty} E_{0 \epsilon_{n}}=\lim _{n \rightarrow \infty} E_{0 \epsilon_{n}}
$$

we have $E_{0} y=y$ and $E_{0} z=0$ by (3.3), (3.4) and Lemma 3.1. Thus we may take $E_{y z}=E_{0}$. This completes the proof.

3.5. Corollary. A bounded Boolean algebra of projections in a reflexive space is complete if and only if it is strongly closed.

4. Weakly closed algebras. The theorems of $\S \S 2$ and 3 enable us to prove the following result. 
4.1. THEOREM. Let $B$ be a bounded Boolean algebra of projections in a reflexive space, and let $\mathbb{W}$ be the weakly closed algebra generated by $\mathfrak{B}$. Then $\mathbb{B}$ is generated in the uniform topology by $\bar{B}$. Each operator in $\mathbb{B}$ is a scalar type spectral operator whose resolution of the identity has its range in $\overline{B^{s}}$.

Proof. Let $A$ be an element of $\mathfrak{S}$. Then since $\mathfrak{B}=\overline{\mathscr{Q}}^{w}=\overline{\mathfrak{Q}}^{s}$ there is a net $\left\{A_{a}\right\} \subseteq \mathscr{U}$ such that $A x=\lim _{a} A_{a} x, x \in \mathfrak{X}$. Let $A_{\alpha}=S\left(f_{a}\right), f_{a} \in C(\mathbb{M})$. Now $f_{\alpha}=g_{\alpha}+i h_{\alpha}$ where $g_{\alpha}$ and $h_{\alpha}$ are real, and $A_{\alpha}=B_{\alpha}+i C_{\alpha}, B_{\alpha}=S\left(g_{\alpha}\right), C_{\alpha}=$ $S\left(h_{\alpha}\right)$. Moreover,

$$
B_{\alpha} x-B_{\beta} x=\int_{\mathfrak{M}} r_{\alpha_{\beta}}(m) E(d m)\left(A_{\alpha} x-A_{\beta} x\right)
$$

where

$$
r_{\alpha \beta}(m)=\left\{\begin{array}{l}
\frac{g_{\alpha}(m)-g_{\beta}(m)}{f_{\alpha}(m)-f_{\beta}(m)}, \\
0,
\end{array}\right.
$$

$$
\begin{aligned}
& f_{\alpha}(m) \neq f_{\beta}(m) \\
& f_{\alpha}(m)=f_{\beta}(m) .
\end{aligned}
$$

Since $\left|r_{\alpha \beta}(m)\right| \leq 1$,

$$
\left|\int_{\mathbb{M}} r_{\alpha \beta}(m) E(d m)\right| \leq 4 M
$$

Thus $\left\{B_{\alpha} x\right\}$ is a Cauchy net for each $x \in \mathfrak{X}$. The operator $B$ defined by

$$
B x=\lim _{a} B_{\alpha} x, \quad x \in \mathfrak{X}
$$

is in $\mathscr{W}$ since the inequality

$$
|B x| \leq 4 M \varlimsup_{a}\left|A_{\alpha} x\right|=4 M|A x|
$$

shows $B$ is bounded. Similarly the net $\left\{C_{\alpha}\right\}$ converges strongly to a bounded operator $C \in \mathfrak{B}$, and $A=B+i C$.

By Theorem $2.3 B$ and $C$ are scalar type spectral operators. To show that $A$ is a scalar type spectral operator it is sufficient to prove that the resolutions of the identity of $B$ and $C$ generate a bounded Boolean algebra. ${ }^{2}$ It will be

2 Cf. [9, Theorem 19]. It is not known whether in a reflexive space the sum of two commuting scalar type spectral operators is a scalar type spectral operator. An example to the contrary has been given by Kakutani $[13]$ in a non reflexive space. 
shown that $E(\cdot ; B)$ and $E(\cdot ; C)$ have their range in $\bar{B} s$. Let $\sigma$ be any bounded closed subset of the real line and let $\left\{\phi_{n}\right\}$ be a monotone decreasing sequence of continuous functions with

$$
\lim _{n \rightarrow \infty} \phi_{n}(\lambda)=k_{\sigma}(\lambda), \quad-\infty<\lambda<\infty .
$$

by Theorem $2.6 \phi_{n}(B) \in \mathbb{W}$ for each $n$. But $\phi_{n}(B)$ converges weakly to $E(\sigma ; B)$, and thus $E(\sigma ; B) \subseteq \mathfrak{W}$. But then $E(\sigma ; B) \in \overline{\mathbb{B}} s$ by Theorem 3.4. The assertion that the range of $E(\cdot ; B)$ is in $\bar{B} s$ now follows from the countable additivity of $E(\cdot ; B) x, x \in X$. The operator $C$ is treated in the same way. Theorem 1.1 may be applied to the bounded Boolean algebra $\bar{B} s$ to conplete the proof.

4.2. COROLLARY. In a reflexive B-space the uniformly closed algebra generated by a complete bounded Boolean algebra of projections is weakly closed.

4.3. REMARK. The use of Theorem 3.4 in the proof of Theorem 4.1 to show $E(\cdot ; B) \subseteq \overline{B^{S}} s$ can be avoided if $X$ is separable. In this case $\sigma(B)$ contains at most denumerabily many eigenvalues, and Theorem 2.6 shows

$$
\lim _{\alpha} E\left((-\infty, \lambda] ; B_{\alpha}\right) x=E((-\infty, \lambda] ; B) x, \quad x \in \mathfrak{X}
$$

for a dense set of numbers $\lambda$.

4.4. Definition. A scalar type spectral operator will be said to be real if $\sigma(A)$ is real.

Our next objective is to characterize the weakly closed algebra generated by a single real scalar type operator and the identity. We will require certain preliminary material.

4.5. Definition. A compact liausdorff space $\Omega$ is extremely disconnected if the closure of every open set is open. A positive regular Borel measure $\mu$ on $\Omega$ is normal if it vanishes on sets of the first category. An extremely disconnected compact Hausdorff space $\Omega$ is hyperstonian if it has sufficiently many normal measures that the union of their supports is dense in $\Omega$.

Stone has shown [26] that the representation space of a complete Boolean algebra is characterized by the property of being extremely disconnected. It can be shown [10] that corresponding to each Borel set $e$ of an extremely disconnected space there is a unique open and closed set $\sigma$ such that the symmetric difference $(e-\sigma) \cup(\sigma-e)$ is of the first category. The notion of a hyperstonian 
space is due to Dixmier [6] who has proved that a compact Hausdorff space is the space of maximal ideals for a commutative $W^{*}$-algebra on Hilbert space if and only if it is hyperstonian. A hyperstonian space $\Omega$ is of countable type if each mutually disjoint family of open and closed subsets of $\Omega$ is at most countable. By a theorem of Dixmier [6] each hyperstonian space contains a family $\Omega_{i}, i \in I$ of mutually disjoint open and closed subsets whose union is dense, with the property that each $\Omega_{i}$ is of countable type.

Now let $\mathscr{B}$ be a complete bounded Boolean algebra of projections in a reflexive space with representation space $\mathfrak{M}$. If $x \in \mathfrak{X}, x^{*} \in \mathfrak{X}^{*}$, the measure $x^{*} E(\cdot) x$ on $\mathbb{M}$ vanishes on sets of the first category. Thus the positive measure $v\left(\cdot ; x^{*}, x\right)$ defined by

$$
v\left(\sigma ; x^{*}, x\right)=\underset{\sigma}{\text { tot. var. }} x^{*} E(\sigma) x
$$

is normal. Clearly the union of the carriers of such measures is dense in $\mathbb{M}$. Following Segal [22] we call a projection $E \in B$ countably decomposable if each mutually disjoint family of projections in $B$ bounded by $E$ is at most countable. Thus we have proved:

4.6. ThEOREM. A complete bounded Boolean algebra of projections in a reflexive space contains a family of mutually disjoint countably decomposable projections whose least upper bound is the identity.

4.7. Definition. Let $A$ be a real scalar type operator. We denote by S $(A)$ the weakly closed algebra generated by $A$ and $I$. An operator $B$ is an extended bounded Baire function of $A$ if for every countably decomposable projection $E$ in $\mathscr{B}(A), B$ commutes with $E$ and the contraction $B_{E}$ of $B$ to $E X$ is a bounded Baire function (in the usual sense) of the contraction $A_{E}$ of $A$.

The concept of an extended bounded Baire function is due to Segal [22]. One verifies easily that the contraction of $A$ to $E X$ is a real scalar type spectral operator.

4.8. THE OREM. The algebra $\mathbb{S}_{(}(A)$ generated in the weak operator topology by a real scalar type operator $A$ and 1 consists of all extended bounded Baire functions of $A$.

Note that since $A$ is real $\mathscr{N}(A)$ is also the weakly closed algebra generated by the resolution of the identity $E(\cdot ; A)$ of $A$. (Cf. the discussion in the proof of Theorem 4.1.) Let $B$ be the Boolean algebra of projections in $\mathscr{B}(A)$. Then $E(\cdot ; A)$ is strongly dense in $B$ by Theorem 4.1. Clearly each extended bounded 
Baire function of $A$ lies in $\mathfrak{u}(A)$ since it lies in the uniformly closed span of $B$ by (1.3). Conversely, let $B \in \mathfrak{W}(A)$ and let $E \in \mathfrak{M}_{i}(A)$ be countably decomposable. Since $B_{E}$ is in $\left.A_{E}\right)$ it is sufficient for the rest of the proof to suppose the identity $I$ is countably decomposable. We next show that the algebra $\mathcal{U}$ generated in the uniform operator topology by $E(\cdot ; A)$ consists of all bounded Baire functions of $A$. If

$$
f=\sum_{i=1}^{n} \alpha_{i} k_{\mu_{i}}
$$

where the sets $\mu_{i}$ are disjoint Baire sets, then

$$
f(A)=\sum_{i=1}^{n} \alpha_{i} E\left(\mu_{i}\right) .
$$

If $E\left(\mu_{j}\right) x=x,|x|=1$, then $|f(A) x|=\left|c_{i j}\right|$. Thus the inequality

$$
E(\cdot, A)-\text { ess } \sup |f(\lambda)|=|f(A)| \leq 4.1 / E(\cdot, A)-\text { ess sup }|f(\lambda)|)
$$

is established for finitely valued functions. From this follows a result of Dunford [ 9; Theorem 15] that $\mathcal{U}$ is equivalent to the algebra of all $E(\bullet, A)-$ essentially bounded Baire functions on $\sigma(A)$. But each bounded Baire function is a uniform limit of finitely valued functions. It remains to show $\mathscr{U}$ is weakly closed. However, this follows from Corollary 4.2 and the next lemma (which is valid for arbitrary Boolean algebras ).

4.9. Lemma. A $\sigma$-complete bounded Boolean algebra of projections in a reflexive space with the property that each mutually disjoint subset is countable is complete.

If $B$ is not complete it contains a monotone net whose least upper bound does not belong to $\mathcal{B}$. By transfinite induction one may construct from the net a family of mutually disjoint projections of cardinality $>\boldsymbol{N}_{0}$.

Theorem 4.8 is due to von Neumann [27] for the case of a self adjoint operator on a separable Hilbert space. The generalization to the case of an arbitrary Hilbert space is proved by Segal [22] as a corollary of his treatment of multiplicity theory. Our proof of Theorem 4.8, via Corollary 4.2, yields a more direct proof of Segal's theorem.

It is important to know when an algebra $\mathfrak{W}$ is $\mathfrak{Z}(A)$ for some $A \in \mathfrak{W}$. An answer is given by the following theorem. 
4.10. THE OREM. For a bounded Boolean algebra $\mathfrak{B}$ of projections in a reflexive space the following conditions are equivalent.

(a). $B$ is separable in the strong operator topology.

(b). $\mathscr{S}_{\mathrm{S}}$ is separable in the weak operator topology.

(c). $\mathbb{B}$ is generated in the weak operator topology by an element $A \in \mathbb{S}$ and the identity.

Clearly (c) implies (b). Let $\left\{A_{n}\right\}$ be weakly dense in $\mathfrak{W}$. Since each $A_{n}$ may be approximated in the uniform topology by a linear combination of projections, there is a sequence $\left\{E_{m}\right\} \subseteq \mathbb{B}$ which generates $\mathfrak{B}$ in the weak topology. By Theorems 4.1 and 3.4 the countable Boolean algebra $\mathfrak{B}_{0}$ generated by $\left\{E_{m}\right\}$ is strongly dense in $B$, proving (a).

The proof that (a) implies ( c) follows a well known argument. Let $\mathfrak{U}_{0}$ be the algebra generated by $\mathfrak{B}_{0}$ in the uniform operator topology and let $\mathbb{M}_{0}$ be its space of maximal ideals. By a theorem of Gelfand [11] $\mathbb{M}_{0}$ is separable metric. Since $\mathrm{M}_{0}$ is totally disconnected, it is homeomorphic to a subset of the Cantor discontinuum $[2 ; \mathrm{p} .121]$, and thus $C\left(\Re_{0}\right)$ contains a real function $h$ which distinguishes points in $\mathbb{M}_{0}$. By the Stone-Weierstrass theorem [25] and Theorem $1.1, A=S(h)$ and $I$ generate $\mathfrak{U}_{0}$. But $\mathfrak{U}_{0}$ is weakly dense in $\mathfrak{W}_{\text {. }}$

When $\chi$ is separable every subset of $B(X)$ is separable in the strong operator topology. This fact for Hilbert space is due to von Neumann [27]. However, the proof in $[20$, p. 12] extends in a natural way to any Banach space.

\section{REFERENCES}

1. L. Alaoglu, Weak topologies of normed linear spaces, Ann. of Math. 41 (1940), $252-267$.

2. P. Alexandroff and H. Hopf, Topologie I, Berlin, 1935.

3. J. Y. Barry, On the convergence of ordered sets of projections, Proc. Amer. Math. Soc. 5 (1954), $313-314$.

4. J. Dieudonné, Sur le Théorème de Hahn-Banach, Revue Sci. 79 (1941), 642-643.

5. J. Dixmier, Les fonctionnelles linéares sur l'ensemble des opérateurs bornés d'un espace de Hilbert, Ann. of Math. 51 (1950), 387-408.

6. - Sur certains espaces considérés par M. H. Stone, Summa Brasiliensis Mathematicae 2 (1951), 151 - 182.

7. N. Dunford, Direct decomposition of Banach spaces, Boletin de la Sociedad Matematica Mexicana 3 (1946), 1-12.

8. (1952), 559-614. 
9. N. Dunford, Spectral operators, Pacific J. Math. 4 (1954), 321 - 354.

10. J. M. G. Fell and J. L. Kelley, An algebra of unbounded operators, Proc. Nat. Acad. Sci. 38 (1952), 592-598.

11. I. Gelfand, Normierte Ringe, Rec. Math. (Math. Sbornik) N.S. 9 (1941), 3-24.

12. F. Hartogs, and A. Rosenthal, Über Folgen analytischer Funktionen. (Ergänzung zur Arbeit im 100. Band), Math. Ann. 104 (1931), 606-610.

13. S. Kakutani, An example concerning uniform boundedness of spectral measures, Pacific J. Math. 4 (1954), $363-372$.

14. I. Kaplansky, A theorem on rings of operators, Pacific J. Math. 1 (1951), 227-232.

15. J. L. Kelley, Convergence in topology, Duke Math. J. 17 (1950), 277-284.

16. M. Lavrentieff, Sur les fonctions d'une variable complexe représentables par des séries de polynomes, Actualités Sci. Ind. 441, Paris, 1936.

17. E. R. Lorch, On a calculus of operators in reflexive vector spaces, Trans. Amer. Math. Soc. 45 (1939), 217-234.

18. S. Mergelyan, On the representation of functions by series of polynomials on closed sets, Doklady Akad. Nauk SSSR (N.S.) 78 (1951), 405-408. [Aner. Math. Soc. Translation No. 85.]

19. E. A. Michael, Transformations from a linear space with weak topology, Proc. Amer. Math. Soc. 3 (1952), 671-676.

20. B.v.Sz. Nagy, Spektraldarstellung linearer Transformationen des Hilbertschen Raumes, Berlin, 1942.

21. F. Rellich, Störungstheorie der Spektralzerlegung II, Math. Ann. 113 (1936), $667-685$.

22. I. E. Segal, Decomposition of operator algebras II: Multiplicity theory, Mem. Amer. Math. Soc. 9 (1951).

23. M. H. Stone, Linear transformations in Hilbert space and their applications to analysis, Amer. Math. Soc. Coll. Publications, vol. 15, New York, 1932.

24. — A general theory of spectra I, Proc. Nat. Acad. Sci. 27 (1941), 83-87.

25. - The generalized Weierstrass approximation theorem, Math. Mag. 21 (1948), $167-184,237-254$.

26. , Boundedness properties in function-lattices, Canadian J. of Math. 1 (1949), 176- 186.

27. J. von Neumann, Zur Algebra der Funktionaloperatoren und Theorie der normalen Operatoren, Math. Ann. 102 (1929), 370-427.

YALE UNIVERSITY 



\section{PACIFIC JOURNAL OF MATHEMATICS}

\section{EDITORS}

\author{
M.M. SCHIFFE R* \\ Stanford University \\ Stanford, California \\ E. HEWITT \\ University of Washington \\ Seattle 5, Washington
}

R.P. DILWORTH

California Institute of Technology Pasadena 4, California

E.F. BECKENBACH**

University of California

Los Angeles 24, California

\section{ASSOCIATE EDITORS}

$\begin{array}{llll}\text { H. BUSEMANN } & \text { P.R. HALMOS } & \text { BORGE JESSEN } & \text { J. J. STOKER } \\ \text { HERBERT FEDERER } & \text { HEINZ HOPF } & \text { PAUL LÉVY } & \text { E.G. STRAUS } \\ \text { MARSHALL HALL } & \text { R.D. JAMES } & \text { GEORGE PÓLYA } & \text { KÔSAKU YOSIDA }\end{array}$

\section{SPONSORS}

UNIVERSITY OF BRITISH COLUMBIA CALIFORNIA INSTITUTE OF TECHNOLOGY UNIVERSITY OF CALIFORNIA, BERKELEY UNIVERSITY OF CALIFORNIA, DAVIS UNIVERSITY OF CALIFORNIA, LOS ANGELES UNIVERSITY OF CALIFORNIA, SANTA BARBARA UNIVERSITY OF NEVADA OREGON STATE COLLEGE UNIVERSITY OF OREGON
UNIVERSITY OF SOUTHERN CALIFORNIA STANFORD RESEARCH INSTITUTE STANFORD UNIVERSITY WASHINGTON STATE COLLEGE UNIVERSITY OF WASHINGTON

AMERICAN MATHEMATICAL SOCIETY HUGHES AIRCRAFT COMPANY

Mathematical papers intended for publication in the Pacific Journal of Mathematics should be typewritten (double spaced), and the author should keep a complete copy. Manuscripts may be sent to any of the editors. Manuscripts intended for the outgoing editors should be sent to their successors. All other communications to the editors should be addressed to the managing editor, E.G. Straus, at the University of California Los Angeles 24, California.

50 reprints of each article are furnished free of charge; additional copies may be obtained at cost in multiples of 50 .

The Pacific Journal of Mathematics is published quarterly, in March, June, September, and December. The price per volume (4 numbers) is $\$ 12.00$; single issues, $\$ 3.50$; back numbers (Volumes $1,2,3$ ) are available at $\$ 2.50$ per copy. Special price to individual faculty members of supporting institutions and to individual members of the American Mathematical Society: $\$ 4.00$ per volume; single issues, $\$ 1.25$.

Subscriptions, orders for back numbers, and changes of address should be sent to the publishers, University of California Press, Berkeley 4, California.

Printed at Ann Arbor, Michigan. Entered as second class matter at the Post Office, Berkeley, California.

* To be succeeded in 1955, by H.L. Royden, Stanford University, Stanford, California.

** To be succeeded in 1955, by E.G. Straus, University of California, Los Angeles 24, Calif.

UNIVERSITY OF CALIFORNIA PRESS - BERKELEY AND LOS ANGELES 


\section{Pacific Journal of Mathematics \\ Vol. 4, No. $3 \quad$ July, 1954}

Nelson Dunford, Spectral operators ........................ 321

John Wermer, Commuting spectral measures on Hilbert space.......... 355

Shizuo Kakutani, An example concerning uniform boundedness of spectral measures...................................... 363

William George Bade, Unbounded spectral operators .............. 373

William George Bade, Weak and strong limits of spectral operators ...... 393

Jacob T. Schwartz, Perturbations of spectral operators, and applications. I. Bounded perturbations ........................... 415

Mischa Cotlar, On a theorem of Beurling and Kaplansky............... 459

George E. Forsythe, Asymptotic lower bounds for the frequencies of certain polygonal membranes ............................... 467 